\title{
SUBSEXPL: A tool for Simulating and Comparing Explicit Substitutions Calculi
}

\author{
A Tutorial \\ Flávio L. C. de Moura* ${ }^{\dagger} \quad$ Mauricio Ayala-Rincón ${ }^{\dagger} \quad$ Fairouz Kamareddine $^{\ddagger}$
}

April 4, 2006

\begin{abstract}
In this tutorial we present the system SUBSEXPL that is used for simulating and comparing explicit substitutions calculi. This framework was developed in Ocaml, a language of the ML family, and it allows the manipulation of expressions of the $\lambda$-calculus and of several styles of explicit substitutions calculi. Applications of this framework include: the visualisation of the contractions of the $\lambda$-calculus, and of guided one-step reductions and normalisation via each of the associated substitution calculi. Many useful facilities are available: reductions can be easily recorded and stored into files, latex output and useful examples for dealing with, among other things, arithmetic operations and computational operators such as conditionals and repetitions in the $\lambda$-calculus. The current implementation of SUBSEXPL includes treatment of three different calculi of explicit substitutions: the $\lambda \sigma$, the $\lambda s_{e}$, the suspension calculus and a refinement of the suspension calculus called combining suspension calculus which allows for combination of steps of $\beta$-contraction; other explicit substitutions calculi can be easily incorporated into the system. An implementation of the $\eta$-reduction is provided for each of these explicit substitutions calculi. This system has been of great help for systematically comparing explicit substitutions calculi, as well as for understanding properties of explicit substitutions such as the Preservation of Strong Normalisation. In addition, it has been used for teaching basic properties of the $\lambda$-calculus such as: computational adequacy, the importance of de Bruijn's notation and of making explicit substitutions in real implementations based on the $\lambda$-calculus.
\end{abstract}

Keywords: $\lambda$-Calculus, Explicit Substitutions, Visualisation of $\beta$ - and $\eta$-Contraction and Normalisation.

\section{Introduction}

In the last decade, a number of explicit substitutions calculi have been developed. Most of these calculi have been claimed to be useful for practical notions such as the implementation of typed functional programming languages and higher-order proof assistants. We describe SUBSEXPL, a system developed in Ocaml, a language of the ML family, which allows for the manipulation of expressions of the $\lambda$-calculus and of three different calculi of explicit substitutions:

1. The $\lambda \sigma$-style [ACCL91] which introduces two different sets of entities: one for terms and one for substitutions.

\footnotetext{
${ }^{*}$ Corresponding author.

${ }^{\dagger}$ Departamento de Matemática, Universidade de Brasília, 70900-910 Brasília D.F., Brasil. \{flavio, ayala\}@mat.unb.br

${ }^{\ddagger}$ School of Mathematical and Computer Sciences, Heriot-Watt University, Edinburgh EH14 4AS, Scotland. fairouz@macs.hw.ac.uk
} 
2. The $\lambda s$-style [KR95] which is based on the philosophy of de Bruijn's Automath [NGdV94] elaborated in the new item notation [KN96]. In this framework, a term is a sequence of items, which can be an application item, an abstraction item, a substitution item or an updating item. The advantages of building the explicit substitutions calculus in this framework include remaining as close as possible to the familiar $\lambda$-calculus (cf. [KR00]).

3. The suspension calculus [Nad99], which introduces three different sets of entities: terms, environments and lists of environments.

4. The "combining suspension calculus" [NaQi03, LNQ04], which is a refinement of the suspension calculus, that allows for combinations of steps of $\beta$-contraction.

Each of these different styles has plus and minus points. Although various attempts have been made at comparing these styles (cf. [AMK05, KR00]), a lot remains to be explained. A better understanding of the similarities and differences of these styles may lead on one hand to solving the remaining open questions related to the various calculi, and on the other hand, to a more inclusive calculus and implementations which combine the advantages in one system.

Through SUBSEXPL, we attempt to understand the working of the rewrite rules of these calculi. We develop a full scale Ocaml implementation of the three calculi where contractions in all these calculi (including those in the type free $\lambda$-calculus) can be visualised in a step-wise fashion and where the behaviour of the reduction paths can be analysed. Especially, we concentrate on the one-step guided reductions and normalisation via each of the associated substitution calculi.

SUBSEXPL has been successfully used for teaching our students basic properties of the $\lambda$-calculus such as: computational adequacy, the importance of de Bruijn's notation and of making explicit substitutions in real implementations based on the $\lambda$-calculus. SUBSEXPL has also been of great importance for systematically comparing these three calculi of explicit substitutions.

Furthermore, SUBSEXPL includes adequate implementations of the rules of $\eta$-reduction for the three calculi as well as a clean implementation for the $\lambda s_{e}$-calculus (cf. [AMK05]) in the sense that no other rewriting rules than the ones strictly involved in the Eta-contraction ${ }^{1}$ are included in one-step Eta-contraction. Work on higher-order unification (HOU) in $\lambda \sigma$ and $\lambda s_{e}$ established the importance of combining Eta-reduction or contraction (as well as expansion) with explicit substitutions. This has provided extensions of $\lambda \sigma$ and $\lambda s_{e}$ with Eta-reduction rules also referred to by $\lambda \sigma$ and $\lambda s_{e}$ (cf.[DHK00, ARK01]). Eta reduction (as well as expansion) is necessary for working with functions and programs, since one needs to express functional or extensional equality, i.e., when the application of two $\lambda$-terms to any term yields the same result, then they should be considered equal. This led to various extensions of explicit substitutions calculi with an Eta rule even before this was applied to HOU [Har92, Río93, Bri95, Kes00].

The output of $\lambda$-terms is a difficult point because $\lambda$-expressions may become big very quickly. In order to ease reading the output of the system, we provided the latex output which can be generated at any step of the reduction and, moreover, the generated file can be easily edited according to the user's requirement.

SUBSEXPL has been used as a tool for understanding properties of explicit substitutions calculi. Desired properties of an explicit substitutions calculus include:

(a) Simulation of one step $\beta$-reduction: whenever $a$ reduces to $b$ in the $\lambda$-calculus using one step $\beta$ reduction, we have that $a$ reduces to $b$ in the explicit substitutions calculus using one step of the explicit $\beta$-reduction (starting rule) and the substitution rules.

(b) Confluence (CR): confluence is the property that establishes that reductions do not depend on reduction strategies or in other words, that whenever a term can be reduced in two different ways, the obtained terms can be joined by rewriting into a common term. CR is considered for two classes of terms:

(b.1) Ground terms: these are the usual terms of the $\lambda$-calculus built from variables, applications and abstractions.

\footnotetext{
${ }^{1}$ We use the Greek letter $\eta$ to refer only to the " $\eta$-rule" of the pure $\lambda$-calculus, and its name "Eta" to refer to the corresponding rule in the explicit substitutions calculi.
} 
(b.2) Open terms: in this case, the language of the explicit substitutions calculus is expanded with a new class of variables, known as meta-variables. In this setting, open terms can be seen as contexts and meta-variables as place-holders. Open terms are essential in higher-order unification algorithms that uses explicit substitutions [DHK00, ARK01].

(c) Strong normalisation (SN) of the underlying calculus of explicit substitutions: this property asserts about the termination of the explicit substitutions calculi without the explicit $\beta$-reduction rule; i.e., without the rule that starts the simulation of the $\beta$-reduction.

(d) Preservation of SN (PSN): whenever all possible reductions starting from a pure $\lambda$-term are terminating in the $\lambda$-calculus, there are no possible infinite reductions starting from this term in the explicit substitutions calculus.

Without Eta, $\lambda \sigma$ satisfies (a), (b.1), (c) and satisfies (b.2) only when the set of open terms is restricted to those which admit meta-variables of sort terms. Without Eta, $\lambda s$ satisfies (a)..(d) but not (b.2). However, $\lambda s$ has an extension $\lambda s_{e}$ (again without Eta) for which (a), (b.1) and (b.2) holds, but (d) fails and (c) is unknown. The suspension calculus (which does not have Eta) satisfies (a) and when restricted to well formed terms it also satisfies (b.1), (b.2) and (c), but (d) is unknown.

SUBSEXPL has been used as a tool for examining the PSN property of two of the three calculi we consider. The system allows us to follow the counter-examples of Melliès ([Mel95]) and Guillaume ([Gui00]) for proving that neither $\lambda \sigma$ - nor $\lambda s_{e}$-calculi preserve SN. By examining these counter-examples in our system, firstly, one can animate the generation of an infinite derivation in the substitution calculi starting from a well typed term of the pure $\lambda$-calculus; afterwards, one can try to generate infinite derivations of $\beta$-reductions from these $\lambda$ terms; finally, one can conclude that this is impossible. In this way it is possible to simultaneously understand the importance of the PSN property as well as why it does not hold in these two calculi. The animated generation of the initial steps of these infinite derivations inside each calculus gives an easy-to-understand and more intuitive insight about why the PSN property fails. Consequently, we believe SUBSEXPL is an adequate and useful tool for intuitively understanding the details and difficulties concerned with general properties of the $\lambda$-calculus as well as of explicit substitutions calculi.

In section 2 we briefly describe the system and its usage. In section 3 we present the applications of the system.

\section{Description of SUBSEXPL}

SUBSEXPL is an implementation of the rewriting rules of the three treated calculi of explicit substitutions. SUBSEXPL is an open source software, runs over GNU/Linux platforms and is available at www. mat . unb. br/ ayala/TCgroup/.

\subsection{General details of the implementation}

SUBSEXPL was developed in OCAML since it provides a natural environment for pattern-matching and rewriting. Moreover, there exists an active OCAML group around the world always ready to collaborate with implementational questions. Since SUBSEXPL does not use specific object tools of Ocaml it is easily adapted to any other language of the ML family such as Caml and SML.

The three explicit substitutions calculi considered in the previous section use $\lambda$-terms in de Bruijn notation, which is important for computational implementations of the $\lambda$-calculus since every $\alpha$-equivalent term has a unique representation in this notation. SUBSEXPL accepts both pure and non pure $\lambda$-terms in de Bruijn notation as input. A pure $\lambda$-term is built solely with applications and abstractions (hence a term of the unextended $\lambda$-calculus). A non pure $\lambda$-term includes some internal operators of one of the explicit substitutions calculi. The terms of the three calculi are implemented in SUBSEXPL as follows: 


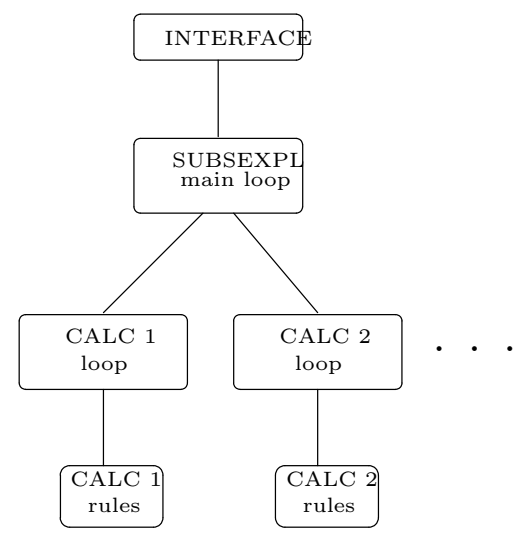

Figure 1: Structure of the system SUBSEXPL.

1. $\lambda \sigma$-terms of the form $\underline{1}, \lambda M,(M N)$ and $M[S]$ are respectively represented as One, L (M), A (M, N) and $\mathrm{Sb}(\mathrm{M}, \mathrm{S}) ; \lambda \sigma$-substitutions of the form $i d, \uparrow, M . S$ and $S \circ T$ are respectively represented as Id, Up, $\operatorname{Pt}(\mathrm{M}, \mathrm{S})$ and $\mathrm{Cp}(\mathrm{S}, \mathrm{T})$.

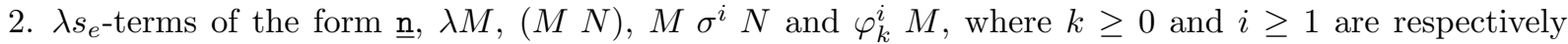
represented as $n, L(M), A(M, N), S(i, M, N)$ and $P(i, k, M)$.

3. $\lambda_{\mathrm{SuSP}}$-terms of the form $\underline{\mathrm{n}}, \lambda M,(M N)$ and $\llbracket M, i, j, e_{1} \rrbracket$ are respectively represented as $\mathrm{n}, \mathrm{L}(\mathrm{M}), \mathrm{A}(\mathrm{M}, \mathrm{N})$ and $\operatorname{Sp}(\mathrm{M}, i, j, e 1) ; \lambda_{\mathrm{SuSP}}$-environments of the form $n i l$, et :: $e_{1}$ and $\left\{\left\{e_{1}, i, j, e_{2}\right\}\right.$ as Nil, Con (et,e1) and $\mathrm{Ck}(\mathrm{e} 1, \mathrm{i}, \mathrm{j}, \mathrm{e} 2) ; \lambda_{\mathrm{SusP}}$-environments terms of the form $@ i,(M, i)$ and $\left\langle\left\langle e t, i, j, e_{1}\right\rangle\right\rangle$ are respectively represented as Ar i, Paar $(M, i)$ and $L G(e t, i, j, e 1)$.

4. Since the combining suspension calculus is a refinement of the suspension calculus, its terms are essentially $\lambda_{\mathrm{Susp}}$-term as above without environments of the form $\left\{\left\{e_{1}, i, j, e_{2}\right\}\right\}$ and without environments terms of the form $\left\langle\left\langle e t, i, j, e_{1}\right\rangle\right\rangle$.

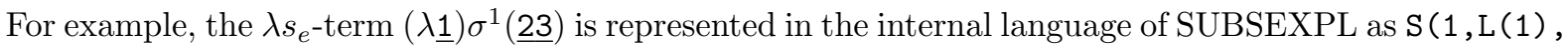
$A(2,3))$. The system allows, for each calculus, the generation of a latex output given together with the original grammar.

The current structure of the system is intended to allow a simpler way to include new calculi. The general structure of the system is represented in Figure 1. A stepwise description on how to include a new calculi can be found in the file adding-a-new-calculi distributed with the source code of the system. A more detailed description of the dependencies of the files in the current implementation is given in Figure 2; the complete documentation related to each of these files can be found in the doc directory which is included in the file that contains the source code of the system.

For each implemented calculi, there are two main parts: matching and reduction. The matching part is responsible for collecting all the existing redexes, for each rewriting rule of the calculus, of the current $\lambda$-term and to store the positions of these redexes into a list. The functions responsible for this work are implemented in the files sematch $\square$.ml (where $\square$ ranges over ls, lse and sus for the $\lambda \sigma-, \lambda s_{e^{-}}$and the suspension calculus, respectively). The reduction part contains the implementations of the rewriting rules of the considered calculus. The files with these functions are named sered $\square$.ml (where $\square$ is as above).

\subsection{Use of the system}

Installation instructions are included in the README file distributed with the source code of the system ${ }^{2}$. In fact, there is no installation, since the user can just download the executable file, named subsexpl.bin,

\footnotetext{
${ }^{2}$ The README file is also available for online reading at www.mat.unb.br/ $\sim f l a v i o$
} 


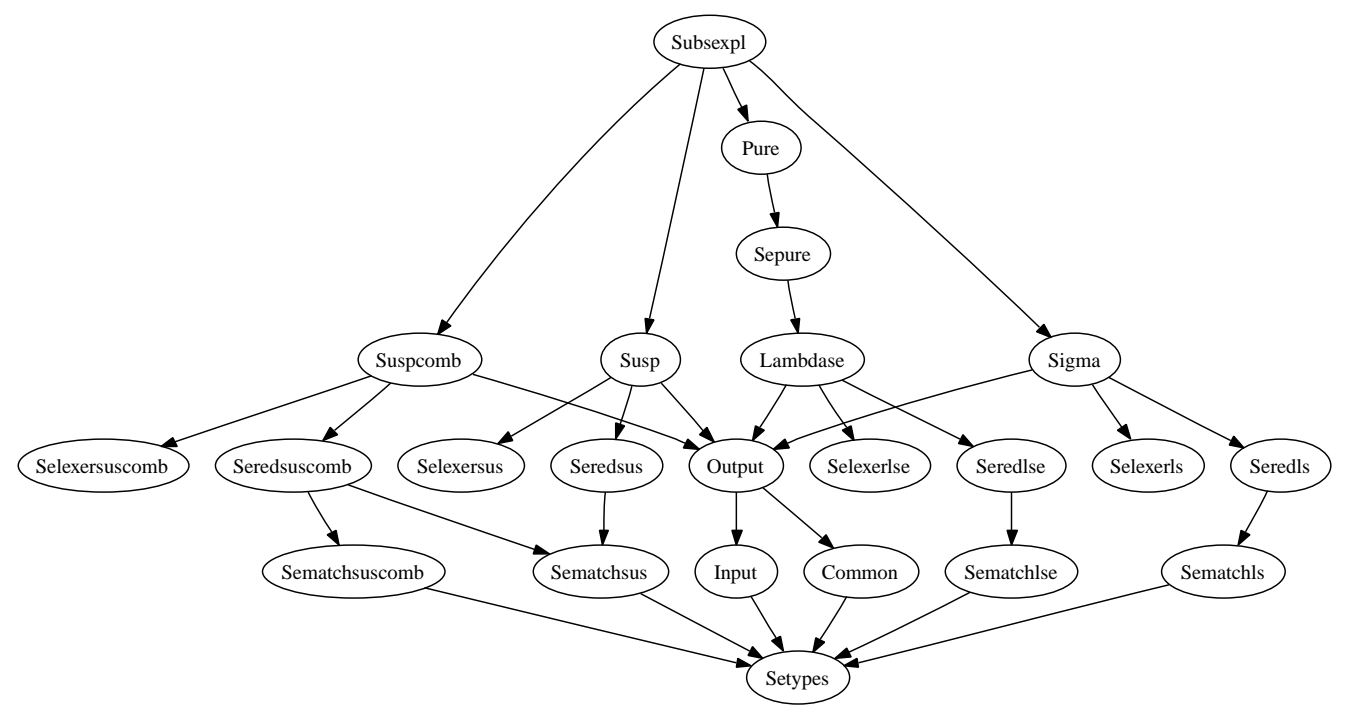

Figure 2: Dependencies amongst the files.

and run the system. Optionally, the user can download the source code of the system, included in the file subsexpl.tar.gz and compile its own executable as explained in the README file. In addition, the file subsexpl.tar.gz also contains some examples which can be used with the system.

To start the system, execute the file subsexpl.bin (by typing ./subsexpl.bin). We recommend the use of the line editor ledit ${ }^{3}$ :

./ledit.out ./subsexpl.bin.

Alternatively, the user can run SUBSEXPL inside a shell in the emacs editor so that (s)he can easily cut and paste and check the balance of expressions. To do so just type within emacs $\mathrm{M}-\mathrm{x}$ shell and then ./subsexpl to start SUBSEXPL.

The first screen is as below where option 4 gives a brief grammatical description of the input and output for each calculus.

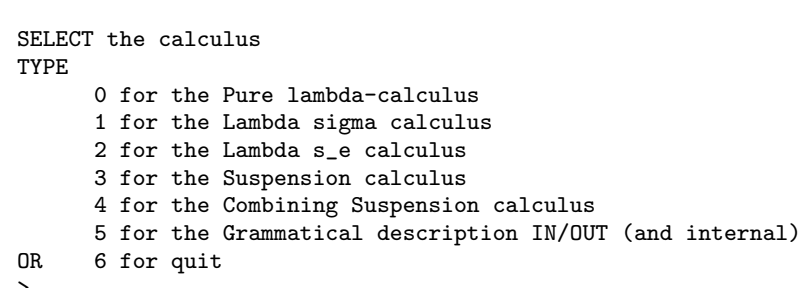

Option 0 allows the user to simulate one-step $\beta$-reduction and $\eta$-reduction as well as normalisations in the pure $\lambda$-calculus, while options 1, 2, 3 and 4 perform simulations of reductions and normalisations in $\lambda \sigma$, $\lambda s_{e}$, suspension calculus and combining suspension calculus, respectively.

As a complete example, we will show how to operate with the Church's numerals (see f. ex.[Bar84]) whose description can be found in the Examples file distributed with the source code. Consider the reduction $A_{+} C_{1} C_{1} \rightarrow{ }_{\beta}^{6} C_{2}$, which evaluates " $1+1$ " in the $\lambda$-calculus, where $A_{+}=\lambda x y p q .((x p)((y p) q))$ and $C_{1}=$ $\lambda f x . f x$. The $A_{+}$operator is written in de Bruijn notation as

$$
A_{+}=\lambda \lambda \lambda \lambda \cdot((\underline{4} \underline{2})(\underline{3} \underline{2}) \underline{1})
$$

\footnotetext{
${ }^{3}$ http://cristal.inria.fr/ ddr
} 
which is translated to the SUBSEXPL language as

$$
L(L(L(L(A(A(4,2), A(A(3,2), 1))))))
$$

Applying this operator to the Church number $C_{1}$, written as $L(L(A(2,1)))$, twice, gives the expression corresponding to $A_{+} C_{1} C_{1}$ in the SUBSEXPL grammar:

$\mathrm{A}(\mathrm{A}(\mathrm{L}(\mathrm{L}(\mathrm{L}(\mathrm{L}(\mathrm{A}(\mathrm{A}(4,2), \mathrm{A}(\mathrm{A}(3,2), 1))))))$,

$\mathrm{L}(\mathrm{L}(\mathrm{A}(2,1)))), \mathrm{L}(\mathrm{L}(\mathrm{A}(2,1))))$

After choosing option 0 in the first screen of the system, we type the above expression:

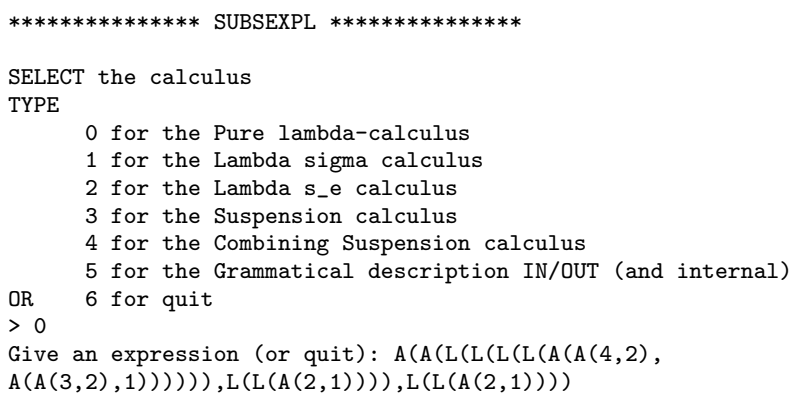

After typing the expression, type ENTER. The next screen will output the current expression and the available redexes for the rules:

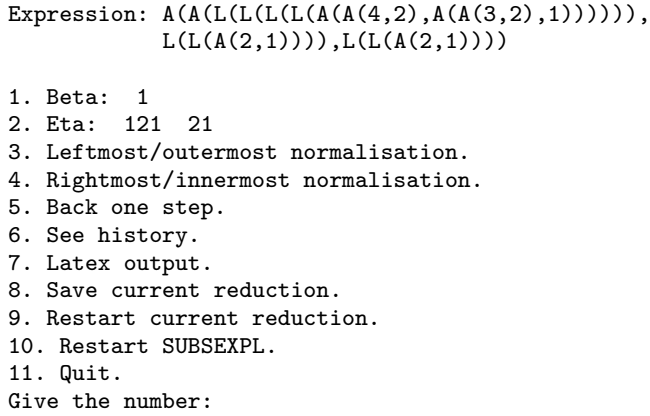

To select $\beta$-reduction, type 1 and then type 1 again to select the redex at position 1 . Now the current screen is:

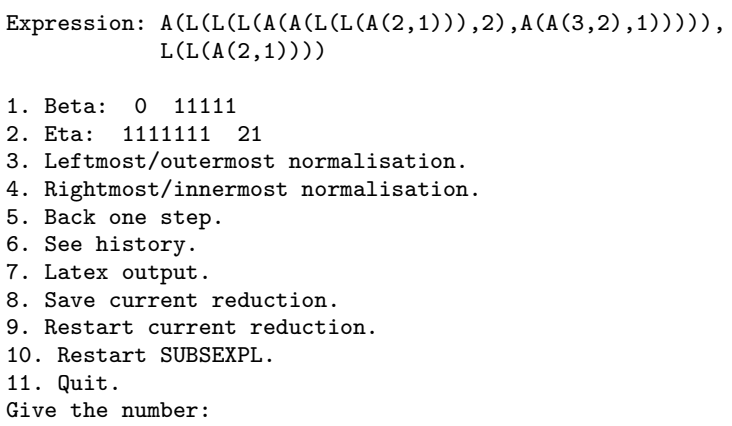

Note that we have two options to apply $\beta$-reduction. One at the root position of the term, written as 0 , and another at position 11111. To reduce the term at position 11111, first type 1 to select Beta and then type the position. Continue the reduction until you get a normal term: 
1. Beta:

2. Eta:

3. Leftmost/outermost normalisation.

4. Rightmost/innermost normalisation.

5. Back one step.

6. See history.

7. Latex output.

8. Save current reduction

9. Restart current reduction.

10. Restart SUBSEXPL.

11. Quit.

Give the number:

The additional options of the system are:

3. Leftmost/outermost normalisation.: Normalises the given term choosing always the leftmost redex.

4. Rightmost/innermost normalisation.: Normalises the given term choosing always the rightmost redex.

5. Back one step: Allows the user to return to the previous step in the current derivation.

6. See history: Shows in the current screen the list of all expressions generated in the current reduction.

7. Latex Output: Generates automatically a file with the latex code of the current reduction and display the dvi file on the screen ${ }^{4}$

8. Save current reduction.: Allows the user to save the current reduction into a simple text file, say my-reduction. To load this reduction the user should restart the system giving this file as argument: ./ledit.out ./subsexpl.bin my-reduction.

9. Restart current reduction: Allows the user to restart the current reduction from the beginning after asking if the user wants to save the current reduction.

10. Restart SUBSEXPL: Restarts the system after asking if the user wants to save the current reduction.

11. Quit: Halts the system after asking if the user wants to save the current reduction.

To generate the latex output, which is possible to be generated even during the intermediate steps in a reduction, just type 7 and then give a file name without any extension. For example, my_file. In this case, the system will generate a dvi file named my_file.dvi. Note that in the latex output, all the redexes you chose during the reduction will appear underlined:

$$
\begin{aligned}
& (((\lambda(\lambda(\lambda(\lambda((\underline{42})((\underline{32}) \underline{1}))))))(\lambda(\lambda(\underline{21}))))(\lambda(\lambda(\underline{21})))) \rightarrow_{\beta} \\
& ((\lambda(\lambda(\lambda(((\lambda(\lambda(\underline{21}))) \underline{2})((\underline{32}) \underline{1})))))(\lambda(\lambda(\underline{21})))) \rightarrow \beta
\end{aligned}
$$

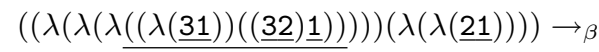

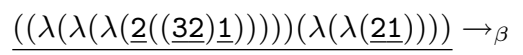

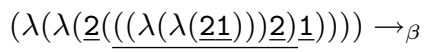

$$
\begin{aligned}
& (\lambda(\lambda(\underline{(2} \underline{((\lambda(\underline{31})) \underline{1})}))) \rightarrow \beta \\
& (\lambda(\lambda(\underline{2}(\underline{21}))))
\end{aligned}
$$

An interesting exercise is to simulate such a derivation step by step using the $\lambda \sigma$, the $\lambda s_{e}$ or the suspension calculus. The current implementation has two normalisation strategies available: the leftmost/outermost strategy or the strategy according to the order of the rules given on the screen of each calculi (we call this strategy 'random'). A interesting fact is that the first step of the previous example when simulated in the $\lambda \sigma$-calculus using the random normalisation strategy generates some huge terms which exceeded the available memory for the latex compilation. In fact, the simulation of the first $\beta$-reduction using the 'random' strategy is done in 236 steps, while the same simulation using the leftmost strategy is performed in only 45 steps! The complete reduction using the leftmost/outermost strategy generated about 3 full pages of latex output with small fonts. In the $\lambda s_{e}$ as well as in the suspension calculus, both strategies generate the output within about 2 pages.

\footnotetext{
${ }^{4} \mathrm{We}$ assume that the running system has latex and xdvi installed.
} 
Terms with internal operators of the explicit substitutions calculi implemented in SUBSEXPL may be given as input: as an example, take the $\lambda \sigma$-term $((\lambda \underline{1}) \underline{1}[\uparrow])[\underline{1} . i d]$ which is written in SUBSEXPL as

$$
\mathrm{Sb}(\mathrm{A}(\mathrm{L}(1), \mathrm{Sb}(\text { One }, \mathrm{Up})), \mathrm{Pt}(\text { One }, \mathrm{Id}))
$$

Giving this term to the system we get the following screen:

Expression: $\mathrm{Sb}(\mathrm{A}(\mathrm{L}(\mathrm{One}), \mathrm{Sb}(\mathrm{One}, \mathrm{Up})), \mathrm{Pt}(\mathrm{One}, \mathrm{Id}))$

1. Beta: 1

2. App: 0

3. Abs:

4. Clos:

5. VarCons:

6. Id:

7. Assoc:

8. Map:

9. IdL:

10. IdR:

11. ShiftCons:

12. VarShift:

13. SCons:

14. Eta:

15. One beta full step (leftmost): 1

16. One beta full step (random): 1

17. Back one step.

18. See history.

19. Latex output.

20. Save current reduction.

21. Restart current reduction.

22. Restart SUBSEXPL.

23. Quit.

Give the number:

And the reduction can be continued as usual.

\subsection{Implementation of Eta contraction}

SUBSEXPL includes implementations of the Eta rule for each of the three calculi of explicit substitutions treated here. The implementation follows the notion of cleanness as defined in [AMK05]. The intuitive idea of a clean Eta implementation is that it does not mix isolated applications of Eta-reduction with applications of other rules of the corresponding substitution calculi that the ones strictly involved in the Eta-reduction. Clean implementations of the Eta rule allow us to reach good simulations of the Eta-contraction, which implies the possibility of combining steps of Beta and Eta contraction between the languages of these calculi.

The suspension calculus did not originally have an Eta-rule. In [AMK05] this calculus was enlarged with an adequate Eta-rule in the so-called $\lambda_{\mathrm{Susp}}$ calculus. For the enlarged calculus $\lambda_{\mathrm{Susp}}, \lambda s_{e}$ and $\lambda \sigma$ we showed that there exists a correspondence among their Eta-rules which means that, when applied to pure $\lambda$-terms, these rules behave similarly (cf. [AMK05]).

Neither the suspension calculus nor the $\lambda \sigma$-calculus has completely clean implementations of the Etarule. In fact, in these calculi, the implementation of the Eta rule requires the application of some rewriting rules, not directly related to Eta contraction, but which are necessary to normalise some simple terms. Nevertheless, our implementation of the Eta-rule for $\lambda s_{e}$ is clean.

Eta-reduction is important to computational problems that arise in applications of the $\lambda$-calculus. For instance, in [DHK00, ARK01] $\eta$-reduction is useful in the treatment of higher order unification via explicit substitutions calculi $\left(\lambda \sigma\right.$ and $\left.\lambda s_{e}\right)$.

\section{Applications}

SUBSEXPL has been successfully used to teach computational notions of the $\lambda$-calculus as well as to compare and understand some properties of explicit substitutions calculi. In this way, SUBSEXPL can be seen as a tool with both educational and research purposes. In this section we start by explaining how the system can 
be used for educational purposes exploring some computability notions over the $\lambda$-calculus. After that, we explain how it can be used to compare calculi of explicit substitutions according to the computational effort necessary to simulate one step of $\beta$-reduction and finally we show how SUBSEXPL can be used to follow the counter-examples of Melliès and Guillaume that establish that the $\lambda \sigma$-and the $\lambda s_{e}$-calculus, respectively, do not preserve strong normalisation.

\subsection{Understanding the $\lambda$-calculus and its implementations}

We have used SUBSEXPL to explain to students questions related to the computational adequacy of the $\lambda$-calculus and the problems which arise from the usual notation with symbolic variables and the implicit notion of substitution. The computational expressiveness of the $\lambda$-calculus can be illustrated by examples which range from the $\lambda$-representation of arithmetic operations such as addition (which we have illustrated earlier in this paper), multiplication and exponentiation over Church's numerals to the $\lambda$-representation of basic data structures which include booleans and computational commands and operators such as if-thenelse, iteration and recursion. All this was done in the spirit of [Bar84, Bar92]. As a concrete example, we consider an expression for computing the factorial function. This simple exercise takes a lot of effort, because students are neither familiar with the notation neither with the operational semantics of the $\lambda$ calculus. But implementing this class of exercises is necessary because this gives the real flavour of the computational power of the $\lambda$-calculus. By using SUBSEXPL over an intelligent editor such as EMACS we can very quickly implement these functions: Initially, we create abbreviations for the needed operators and functions; afterwards, we compound these operators and functions in order to complete the desired function. We illustrate how this is done for the case of the factorial function. Basically, this function is implementing by defining an iteration operator $T_{H}$ given by $\lambda p \cdot\left\langle S^{+}(p\right.$ true $), H(p$ true $)(p$ false $\left.)\right\rangle$, where $S^{+}$is the successor function, i.e., $S^{+}=A_{+} C_{1}$ and $H$ is a convenient function that does the right job. The result of applying $T_{H}$ to $\left\langle C_{i}, C_{f(i)}\right\rangle$ is the pair $\left\langle C_{i+1}, C_{f(i+1)}\right\rangle$, where $f$ references the function implemented by the iteration mechanism, the first component of the pair is a counter for the iteration step and the second one is the value of the desired function at that step. This iteration operator is then used repeatedly.

\subsubsection{Abbreviations}

1. The Church numbers are as given before;

2. The booleans true and false correspond to the $\lambda$-terms $\mathrm{L}(\mathrm{L}(2))$ and $\mathrm{L}(\mathrm{L}(1))$, respectively.

3. $\langle M, N\rangle$ represents the pair operator which is given, in the language of SUBSEXPL, by the $\lambda$-term $\mathrm{L}(\mathrm{A}(\mathrm{A}(1, \mathrm{M}), \mathrm{N}))$. Pairs can be applied to booleans, written as $\langle M, N\rangle$ true and $\langle M, N\rangle$ false and the normal form of these terms are $\mathrm{M}$ and $\mathrm{N}$, respectively.

4. For the case of the factorial function, the adequate operator $\mathrm{T}$ is given as $T_{H}$ above where $H$ is selected as $\lambda x y . A_{*} y\left(S^{+} x\right)$. It is easy to see that this operator satisfies the property: $\mathrm{T}\left\langle C_{k}, C_{k !}\right\rangle \beta$-reduces to $\left\langle C_{k+1}, C_{(k+1) !}\right\rangle$, and so, applying repeatedly this mechanism we are counting the number of iteration in the first component of the pair and computing the associated value of the factorial in second one.

In the language of SUBSEXPL, the normal form of the operator $\mathrm{T}$ for factorial is given by:

$\mathrm{L}(\mathrm{L}(\mathrm{A}(\mathrm{A}(1, \mathrm{~L}(\mathrm{~L}(\mathrm{~A}(2, \mathrm{~A}(\mathrm{~A}(\mathrm{~A}(4, \mathrm{~L}(\mathrm{~L}(2))), 2), 1)))))$,

$\mathrm{L}(\mathrm{A}(\mathrm{A}(3, \mathrm{~L}(\mathrm{~L}(1))), \mathrm{L}(\mathrm{A}(2, \mathrm{~A}(\mathrm{~A}(\mathrm{~A}(4, \mathrm{~L}(\mathrm{~L}(2))), 2), 1))))))))$

\subsubsection{Checking parts of the implementation}

This step is useful for testing the functionality of parts of the intended implementation which allows to infer the functionality of the whole specification. For instance, we can check that $\mathrm{T}\left\langle C_{2}, C_{2 !}\right\rangle$ reduces to $\left\langle C_{3}, C_{3 !}\right\rangle$. In the input syntax of SUBSEXPL this is written as 


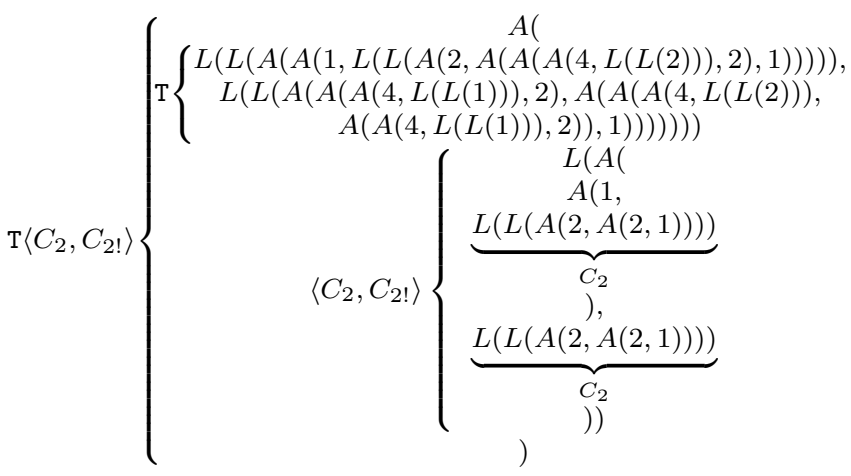

By $\beta$-normalisation this part of the implementation can be checked obtaining the term $\mathrm{L}(\mathrm{A}(\mathrm{A}(1, \mathrm{~L}(\mathrm{~L}(\mathrm{~A}(2, \mathrm{~A}(2, \mathrm{~A}(2,1))))))$, $\mathrm{L}(\mathrm{L}(\mathrm{A}(2, \mathrm{~A}(2, \mathrm{~A}(2, \mathrm{~A}(2, \mathrm{~A}(2, \mathrm{~A}(2,1))))))))))$ which corresponds to $\left\langle C_{3}, C_{3 !}\right\rangle$

The repetition mechanism is completed by applying $n$ times the iteration operator starting from the pair $\left\langle C_{0}, C_{0 !}\right\rangle$. This is done by the term:

$$
A\left(A\left(C_{n}, \mathrm{~T}\right),\left\langle C_{0}, C_{0 !}\right\rangle\right)
$$

which reduces to $\left\langle C_{n}, C_{n !}\right\rangle$.

Functionality of all parts of the desired mechanism/function can be checked by normalisation with SUBSEXPL.

\subsubsection{Final function}

Once enough tests have been ran over SUBSEXPL, the factorial function can be written as:

$$
L(\underbrace{A(\underbrace{A\left(A(1, \mathrm{~T}),\left\langle C_{0}, C_{0 !}\right\rangle\right)}_{\text {Match with eq. }(1)}, \underbrace{L(L(1))}_{\text {false }})}_{\text {Selection of the } 2^{\text {nd }} \text { element of the pair }})
$$

The equation (2), when applied to the Church numeral $C_{n}, \beta$-reduces to $C_{n !}$. In fact, such an application will generate a $\beta$-redex in the root of the new term. Reducing this new term, there is a sub-term of eq. (2) which reduces exactly to the term corresponding to eq. (1). And, this term we have already showed that reduces to the pair $\left\langle C_{n}, C_{n !}\right\rangle$. To get the desired result we need to select the second element of this pair which is done by applying it to false, as previously explained.

Observe that in the syntax of SUBSEXPL (which corresponds to the one of the $\lambda$-calculus) the expression for factorial (eq. (2)) is incomprehensible:

$\mathrm{L}(\mathrm{A}(\mathrm{A}(\mathrm{A}(1, \mathrm{~L}(\mathrm{~L}(\mathrm{~A}(\mathrm{~A}(1, \mathrm{~L}(\mathrm{~L}(\mathrm{~A}(2, \mathrm{~A}(\mathrm{~A}(\mathrm{~A}(4, \mathrm{~L}(\mathrm{~L}(2)))), 2), 1)))))$,

$\mathrm{L}(\mathrm{L}(\mathrm{A}(\mathrm{A}(\mathrm{A}(4, \mathrm{~L}(\mathrm{~L}(1))), 2), \mathrm{A}(\mathrm{A}(\mathrm{A}(4, \mathrm{~L}(\mathrm{~L}(2)))$,

$A(A(4, L(L(1))), 2)), 1)))))))), L(A(A(1, L(L(1)))$,

$L(L(A(2,1)))))), L(L(1))))$

Similarly, other functions can be implemented easily. In fact, notice that from this construction it is easy (also for students) to infer that the sole thing to be changed in the whole repetition mechanism is the function $H$ in the definition of the iteration operator $T_{H}$. For instance, for computing the function $\sum_{i=0}^{n} i$, $H$ should be replaced by $\lambda x y \cdot A_{+} y\left(S^{+} x\right)$; for computing the function $\sum_{i=0}^{n} i^{2}, H$ should be replaced by $\lambda x y \cdot A_{+} y\left(A_{*}\left(S^{+} x\right)\left(S^{+} x\right)\right)$; etc.

We believe that this kind of experiments is necessary and useful for obtaining a flavor of the computational power of the $\lambda$-calculus. A way to speed-up the generation of non elementary implementations is by using our system jointly with an editor for creating the necessary abbreviations, cutting, pasting and testing for modular constructions of "programs" or functions. In intelligent editors such as EMACS, these abbreviations 
can be easily incorporated in new buttons and short-cut keys, which makes the quick construction of these functions possible. Some of these experiments are included in the file of examples of the distribution.

\subsection{Comparing calculi by the simulation of $\beta$-reduction}

SUBSEXPL has been implemented with the intention of comparing the three treated calculi of explicit substitutions with respect to the necessary effort to simulate one-step $\beta$-reduction. By applying this system we were able to conclude that $\lambda s_{e}$ is more efficient than the suspension calculus and is incomparable to the $\lambda \sigma$ calculus in the simulation of one-step $\beta$-reduction [AMK05]. The efficiency of $\lambda s_{e}$ is justified by the fact that the manipulation of de Bruijn indexes in $\lambda s_{e}$ is directly related to a built-in manipulation of natural numbers and arithmetic (which is standard in today's computational environments and programming languages) whereas in the other two calculi, this is done constructively. Of course this comparison is interesting, but not conclusive since $\lambda s_{e}$ is not completely adequate for combining steps of $\beta$-reduction, which is more natural in $\lambda_{\text {SUSP }}\left[\right.$ LN02, Nad02]. But we believe this has to be investigated more carefully, since some variations of $\lambda s_{e}$ like $\lambda t([\mathrm{KR} 00])$, which is a calculus à la $\lambda s_{e}$ but which updates à la $\lambda \sigma$, can allow this combination in the $\lambda \sigma$ family of calculi.

\subsection{Understanding properties of explicit substitutions calculi}

SUBSEXPL has been used as a tool for understanding properties of explicit substitutions calculi. This is illustrated by examining the property of Preservation of Strong Normalisation (PSN).

To illustrate the use of SUBSEXPL in understanding properties of explicit substitution calculi, we explain how one can follow(/check) papers which prove some properties of these calculi. In particular, we follow the proofs of non PSN of $\lambda \sigma$ and $\lambda s_{e}$ given in [Mel95] and [Gui00], respectively. By examining these counter-examples in SUBSEXPL, firstly, students can animate the generation of an infinite derivation in the associated substitution calculi starting from a well typed term of the pure $\lambda$-calculus. Secondly, students can try to generate infinite derivations of $\beta$-reductions from these $\lambda$-terms, concluding (the most critical of them) that this is impossible. This last step is achieved without necessarily knowing that there are no infinite $(\beta$-)derivations in the $\lambda$-calculus starting from well typed terms. In this way it is possible to simultaneously understand the importance of the PSN property as well as why it does not hold in these two calculi.

\subsubsection{The counter-example of Melliès}

To follow the counter-example in the $\lambda \sigma$-calculus, consider the well typed pure $\lambda$-term written in de Bruijn's notation as $\lambda((\lambda(\lambda \underline{1})((\lambda \underline{1}) \underline{1}))((\lambda \underline{1}) \underline{1}))$. The corresponding term in the language of SUBSEXPL is given by

$$
\mathrm{L}(\mathrm{A}(\mathrm{L}(\mathrm{A}(\mathrm{L}(1), \mathrm{A}(\mathrm{L}(1), 1))), \mathrm{A}(\mathrm{L}(1), 1)))
$$

The infinite reduction is generated by applying an adequate strategy which mixes rules of the associated calculus $\sigma$ with the rule Beta which initiates the simulation of one step $\beta$-reduction. The whole derivation, with the usual grammar of the $\lambda \sigma$-calculus, is given at the end of this subsection according to the numbering of steps given in the following tables.

\begin{tabular}{||c|c|l||}
\hline \hline STEP & RULE & POSITION \\
\hline 1 & 1 & 111 \\
2 & 1 & 1 \\
3 & 4 & 1 \\
4 & 8 & 12 \\
\hline \hline
\end{tabular}

At this point, $\mathrm{L}(\mathrm{Sb}(1, \operatorname{Cp}(\operatorname{Pt}(A(L(1), 1), I d), \operatorname{Pt}(A(L(1), 1), I d))))$ is the current term. Let us define recursively: 


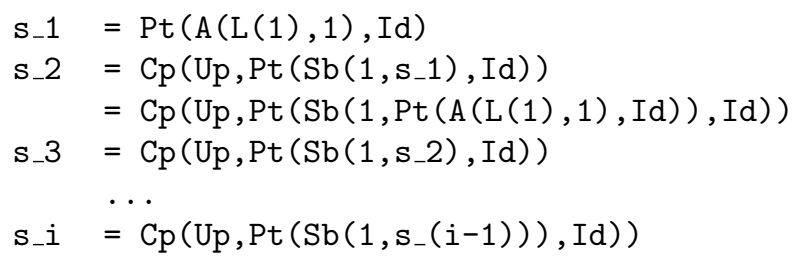

With this definition, we can write the current term as $\mathrm{L}\left(\operatorname{Sb}\left(1, \operatorname{Cp}\left(\mathbf{s}_{-} 1, \mathbf{s}_{-} 1\right)\right)\right)$. At this point, applying the Map transition at position 12 the sub-term $\mathbf{s}_{-} 1$ is duplicated. And we get $\mathrm{L}\left(\operatorname{Sb}\left(1, \operatorname{Pt}\left(\operatorname{Sb}\left(A(L(1), 1), S_{-} 1\right)\right.\right.\right.$, $\left.\left.\left.\mathrm{Cp}\left(\operatorname{Id}, \mathrm{s}_{-} 1\right)\right)\right)\right)$. Note that the second occurrence of $\mathrm{s}_{-} 1$ is vacuous, in the sense that it can be easily eliminated by the rule VarCons. The key idea of Melliès is to maintain this second occurrence of $\mathbf{s}_{-} 1$ and to propagate the first occurrence as follows:

\begin{tabular}{||c|c|l||}
\hline \hline STEP & RULE & POSITION \\
5 & 2 & 121 \\
6 & 9 & 122 \\
7 & 3 & 1211 \\
\hline
\end{tabular}

Now the current term is $\mathrm{L}\left(\mathrm{Sb}\left(1, \mathrm{Pt}\left(\mathrm{A}\left(\mathrm{L}\left(\mathrm{Sb}\left(1, \mathrm{Pt}\left(1, \mathrm{Cp}\left(\mathrm{S}_{-} 1, \mathrm{Up}\right)\right)\right)\right)\right.\right.\right.\right.$, $\left.\left.\left.\mathrm{Sb}\left(1, \mathrm{~S}_{-} 1\right)\right), \mathrm{s}_{-} 1\right)\right)$ ) and again we can apply the Beta rule and then compose the two substitutions:

\begin{tabular}{||c|c|l||}
\hline \hline STEP & RULE & POSITION \\
\hline 8 & 1 & 121 \\
9 & 4 & 121 \\
\hline \hline
\end{tabular}

The next three steps duplicate the sub-term $\operatorname{Pt}(\operatorname{Sb}(1, \operatorname{Pt}(\mathrm{A}(\mathrm{L}(1), 1), \mathrm{Id})), \mathrm{Id})$ and generate the term $\mathrm{s}_{-} 2=\operatorname{Cp}(\operatorname{Up}, \operatorname{Pt}(\operatorname{Sb}(1, \operatorname{Pt}(\mathrm{A}(\mathrm{L}(1), 1), I d)), I d))$ which have inside an occurrence of $\mathbf{s}_{-} 1$ :

\begin{tabular}{||c|c|l||}
\hline \hline STEP & RULE & POSITION \\
\hline 10 & 8 & 1212 \\
11 & 5 & 12121 \\
12 & 7 & 12122 \\
\hline \hline
\end{tabular}

At this point, $\mathrm{L}\left(\mathrm{Sb}\left(1, \mathrm{Pt}\left(\mathrm{Sb}\left(1, \mathrm{Pt}\left(\mathrm{Sb}\left(1, \mathrm{~S}_{-} 1\right)\right.\right.\right.\right.\right.$,

$\left.\left.\left.\left.\mathrm{Cp}\left(\mathbf{s}_{-} 1, \mathbf{s}_{-} 2\right)\right)\right), \mathbf{s}_{-} 1\right)\right)$ ) becomes the current term. It contains an occurrence of $\mathrm{Cp}\left(\mathbf{s}_{-} 1, \mathbf{s}_{-} 2\right)$. By repeating the same sequence of rules, we will get a term with the sub-term $\mathrm{Cp}\left(\mathbf{s}_{-} 2, \mathbf{s}_{-} 3\right)$ :

\begin{tabular}{||c|c|l||}
\hline \hline STEP & RULE & POSITION \\
\hline 13 & 8 & 12122 \\
14 & 2 & 121221 \\
15 & 9 & 121222 \\
16 & 3 & 1212211 \\
17 & 1 & 121221 \\
18 & 4 & 121221 \\
19 & 8 & 1212212 \\
20 & 5 & 12122121 \\
21 & 7 & 12122122 \\
\hline
\end{tabular}

Here, it is easy to see how an infinite reduction can be built from the initial well typed term in the $\lambda \sigma$ calculus of explicit substitutions. The mellies-README file (distributed with the source code) explains these details and some additional information about generating the corresponding postscript file. The above steps are stored in the file mellies and can be executed automatically with the command ./subsexpl.bin mellies (or ./ledit.out ./subsexpl.bin mellies). In this case, the output dvi file, automatically generated by SUBSEXPL, is mellies-ls.dvi. The result can be seen in table 3.3.1. Note that the notation $s_{1}, s_{2}, s_{3}$ and the numeration of the steps are used here for ease reading but is not automatically generated in the above dvi file. The latex code of the output can be found in the file mellies-ls. 


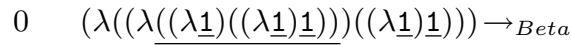

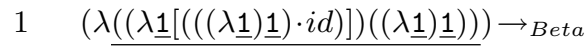

$2 \quad(\lambda \underline{\underline{1}}[(((\lambda \underline{1}) \underline{1}) \cdot i d)][(((\lambda \underline{1}) \underline{1}) \cdot i d)]) \rightarrow C l o s$

$3 \quad\left(\lambda \underline{1}[(\underbrace{(((\lambda \underline{1}) \underline{1}) \cdot i d)} \circ \underbrace{(((\lambda \underline{1}) \underline{1}) \cdot i d))}]) \rightarrow_{M a p}\right.$

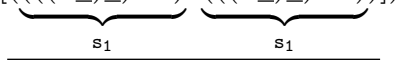

$4 \quad(\lambda \underline{1}[(((\lambda \underline{1}) \underline{1})[(((\lambda \underline{1}) \underline{1}) \cdot i d)] \cdot(i d \circ(((\lambda \underline{1}) \underline{1}) \cdot i d)))]) \rightarrow A_{p p}$

$5 \quad(\lambda \underline{1}[(((\lambda \underline{1})[(((\lambda \underline{1}) \underline{1}) \cdot i d)] \underline{1}[(((\lambda \underline{1}) \underline{1}) \cdot i d)]) \cdot(i d \circ(((\lambda \underline{1}) \underline{1}) \cdot i d)))]) \rightarrow_{I d L}$

$6 \quad(\lambda \underline{1}[(((\lambda \underline{1})[(((\lambda \underline{1}) \underline{1}) \cdot i d)] \underline{1}[(((\lambda \underline{1}) \underline{1}) \cdot i d)]) \cdot(((\lambda \underline{1}) \underline{1}) \cdot i d))]) \rightarrow A b s$

$7 \quad(\lambda \underline{1}[(((\lambda \underline{1}[(\underline{1} \cdot((((\lambda \underline{1}) \underline{1}) \cdot i d) o \uparrow))]) \underline{1}[(((\lambda \underline{1}) \underline{1}) \cdot i d)]) \cdot(((\lambda \underline{1}) \underline{1}) \cdot i d))]) \rightarrow_{\text {Beta }}$

$8 \quad(\lambda \underline{1}[(\underline{\underline{1}}[(\underline{1} \cdot((((\lambda \underline{1}) \underline{1}) \cdot i d)) \uparrow))][(\underline{1}[(((\lambda \underline{1}) \underline{1}) \cdot i d)] \cdot i d)] \cdot(((\lambda \underline{1}) \underline{1}) \cdot i d))]) \rightarrow_{\text {Clos }}$

$9 \quad(\lambda \underline{1}[(\underline{1}[((\underline{1} \cdot((((\lambda \underline{1}) \underline{1}) \cdot i d) \circ \uparrow)) \circ(\underline{1}[(((\lambda \underline{1}) \underline{1}) \cdot i d)] \cdot i d))] \cdot(((\lambda \underline{1}) \underline{1}) \cdot i d))]) \rightarrow_{M a p}$

$10(\lambda \underline{1}[(\underline{1}[(\underline{\underline{1}}[(\underline{1}[(((\lambda \underline{1}) \underline{1}) \cdot i d)] \cdot i d)] \cdot(((((\lambda \underline{1}) \underline{1}) \cdot i d) \circ \uparrow) \circ(\underline{1}[(((\lambda \underline{1}) \underline{1}) \cdot i d)] \cdot i d)))] \cdot(((\lambda \underline{1}) \underline{1}) \cdot i d))]) \rightarrow$ VarCons

$11(\lambda \underline{1}[(\underline{1}[(\underline{1}[(((\lambda \underline{1}) \underline{1}) \cdot i d)] \cdot(((((\lambda \underline{1}) \underline{1}) \cdot i d) \circ \uparrow) \circ(\underline{1}[(((\lambda \underline{1}) \underline{1}) \cdot i d)] \cdot i d)))] \cdot(((\lambda \underline{1}) \underline{1}) \cdot i d))]) \rightarrow A s s o c$

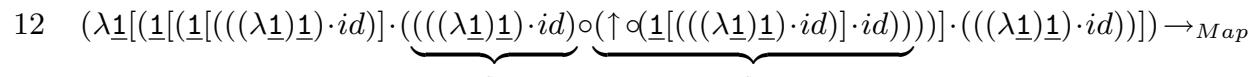

$13(\lambda \underline{1}[(\underline{1}[(\underline{1}[(((\lambda \underline{1}) \underline{1}) \cdot i d)] \cdot(((\lambda \underline{1}) \underline{1})[(\uparrow \circ \underline{1}[(((\lambda \underline{1}) \underline{1}) \cdot i d)] \cdot i d))] \cdot(i d \circ(\uparrow \circ(\underline{1}[(((\lambda \underline{1}) \underline{1}) \cdot i d)] \cdot i d)))))] \cdot(((\lambda \underline{1}) \underline{1}) \cdot i d))]) \rightarrow A p p$

$14 \quad(\lambda \underline{1}[(\underline{1}[(\underline{1}[(((\lambda \underline{1}) \underline{1}) \cdot i d)] \cdot(((\lambda \underline{1})[(\uparrow \circ(\underline{1}[(((\lambda \underline{1}) \underline{1}) \cdot i d)] \cdot i d))] \underline{1}[(\uparrow \circ(\underline{1}[(((\lambda \underline{1}) \underline{1}) \cdot i d)] \cdot i d))])$ $(i d \circ(\uparrow \circ(\underline{1}[(((\lambda \underline{1}) \underline{1}) \cdot i d)] \cdot i d)))))] \cdot(((\lambda \underline{1}) \underline{1}) \cdot i d))]) \rightarrow_{I d L}$

$15 \quad(\lambda \underline{1}[(\underline{1}[(\underline{1}[(((\lambda \underline{1}) \underline{1}) \cdot i d)] \cdot(((\lambda \underline{1})[(\uparrow \circ(\underline{1}[(((\lambda \underline{1}) \underline{1}) \cdot i d)] \cdot i d))] \underline{1}[(\uparrow \circ(\underline{1}[(((\lambda \underline{1}) \underline{1}) \cdot i d)] \cdot i d))]) \cdot$ $(\uparrow o(\underline{1}[(((\lambda \underline{1}) \underline{1}) \cdot i d)] \cdot i d))))] \cdot(((\lambda \underline{1}) \underline{1}) \cdot i d))]) \rightarrow A b s$

$16(\lambda \underline{1}[(\underline{1}[(\underline{1}[(((\lambda \underline{1}) \underline{1}) \cdot i d)] \cdot(((\lambda \underline{1}[(\underline{1} \cdot((\uparrow \circ(\underline{1}[(((\lambda \underline{1}) \underline{1}) \cdot i d)] \cdot i d)) \circ \uparrow))]) \underline{1}[(\uparrow \circ(\underline{1}[(((\lambda \underline{1}) \underline{1}) \cdot i d)] \cdot i d))]) \cdot$ $(\uparrow \mathrm{o}(\underline{1}[(((\lambda \underline{1}) \underline{1}) \cdot i d)] \cdot i d))))] \cdot(((\lambda \underline{1}) \underline{1}) \cdot i d))]) \rightarrow_{B e t a}$

$17(\lambda \underline{1}[(\underline{1}[(\underline{1}[(((\lambda \underline{1}) \underline{1}) \cdot i d)] \cdot(\underline{1}[(\underline{1} \cdot((\uparrow \propto(\underline{1}[(((\lambda \underline{1}) \underline{1}) \cdot i d)] \cdot i d)) \circ \uparrow))][(\underline{1}[(\uparrow \circ(\underline{1}[(((\lambda \underline{1}) \underline{1}) \cdot i d)] \cdot i d))] \cdot i d)]$. $(\uparrow o(\underline{1}[(((\lambda \underline{1}) \underline{1}) \cdot i d)] \cdot i d))))] \cdot(((\lambda \underline{1}) \underline{1}) \cdot i d))]) \rightarrow$ Clos

$18(\lambda \underline{1}[(\underline{1}[(\underline{1}[(((\lambda \underline{1}) \underline{1}) \cdot i d)] \cdot(\underline{1}[((\underline{1} \cdot((\uparrow \circ(\underline{1}[(((\lambda \underline{1}) \underline{1}) \cdot i d)] \cdot i d)) \circ \uparrow)) \circ(\underline{1}[(\uparrow \circ(\underline{1}[(((\lambda \underline{1}) \underline{1}) \cdot i d)] \cdot i d))] \cdot i d))]$. $(\uparrow o(\underline{1}[(((\lambda \underline{1}) \underline{1}) \cdot i d)] \cdot i d))))] \cdot(((\lambda \underline{1}) \underline{1}) \cdot i d))]) \rightarrow_{M a p}$

$19 \quad(\lambda \underline{1}[(\underline{1}[(\underline{1}[(((\lambda \underline{1}) \underline{1}) \cdot i d)] \cdot(\underline{1}[(\underline{1}[(\underline{1}[(\uparrow o(\underline{1}[(((\lambda \underline{1}) \underline{1}) \cdot i d)] \cdot i d))] \cdot i d)] \cdot$ $(((\uparrow \circ \underline{1}[(((\lambda \underline{1}) \underline{1}) \cdot i d)] \cdot i d)) \circ \uparrow) \circ(\underline{1}[(\uparrow \circ(\underline{1}[(((\lambda \underline{1}) \underline{1}) \cdot i d)] \cdot i d))] \cdot i d)))]$. $(\uparrow o(\underline{1}[(((\lambda \underline{1}) \underline{1}) \cdot i d)] \cdot i d))))] \cdot(((\lambda \underline{1}) \underline{1}) \cdot i d))]) \rightarrow$ VarCons

$20 \quad(\lambda \underline{1}[(\underline{1}[(\underline{1}[(((\lambda \underline{1}) \underline{1}) \cdot i d)] \cdot(\underline{1}[(\underline{1}[(\uparrow \propto(\underline{1}[(((\lambda \underline{1}) \underline{1}) \cdot i d)] \cdot i d))]$

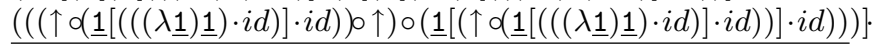
$\overline{(\uparrow o(\underline{1}[(((\lambda \underline{1}) \underline{1}) \cdot i d)] \cdot i d))))] \cdot(((\lambda \underline{1}) \underline{1}) \cdot i d))]) \rightarrow \text { Assoc }}$

$21 \quad(\lambda \underline{1}[(\underline{1}[(\underline{1}[(((\lambda \underline{1}) \underline{1}) \cdot i d)] \cdot(\underline{1}[(\underline{1}[(\uparrow \propto(\underline{1}[(((\lambda \underline{1}) \underline{1}) \cdot i d)] \cdot i d))]$ $((\uparrow \circ \underline{1}[(((\lambda \underline{1}) \underline{1}) \cdot i d)] \cdot i d)) \circ(\uparrow \propto(\underline{1}[(\uparrow \circ(\underline{1}[(((\lambda \underline{1}) \underline{1}) \cdot i d)] \cdot i d))] \cdot i d))))] \cdot(\uparrow \propto(\underline{1}[(((\lambda \underline{1}) \underline{1}) \cdot i d)] \cdot i d))))] \cdot(((\lambda \underline{1}) \underline{1}) \cdot i d))])$ 13

Table 1: The counter-example of Melliès 


\subsubsection{The counter example of Guillaume}

In [Gui00], Guillaume showed that the $\lambda s_{e}$-calculus does not preserve strong normalisation. This is done from the well typed pure $\lambda$-term in de Bruijn's notation:

$$
\lambda((\lambda((\lambda((\lambda \underline{2}) \underline{3})) \underline{2}))(\lambda((\lambda \underline{2}) \underline{2})) \underline{1})
$$

which is written in SUBSEXPL system as

$$
\mathrm{L}(\mathrm{A}(\mathrm{L}(\mathrm{A}(\mathrm{L}(\mathrm{A}(\mathrm{L}(2), 3)), 2)), \mathrm{A}(\mathrm{L}(\mathrm{A}(\mathrm{L}(2), 2)), 1)))
$$

The generation of an infinite derivation is done by the following steps. The initial steps of the infinite derivation, written in the usual grammar of the $\lambda s_{e}$-calculus, is given at the end of this subsection according to the numbering of these steps.

\begin{tabular}{||c|c|l||}
\hline \hline STEP & RULE & POSITION \\
\hline 1 & 1 & 1 \\
2 & 1 & 11 \\
3 & 3 & 11 \\
4 & 2 & 111 \\
5 & 3 & 1 \\
6 & 2 & 11 \\
\hline \hline
\end{tabular}

Here, the derivation gives the sub-term $\mathrm{S}(1, \mathrm{~S}(1,3,2), \mathrm{A}(\mathrm{L}(\mathrm{A}(\mathrm{L}(2), 2)), 1))$ which is called $u_{0}$, and so $u_{0}:=\left(\underline{3} \sigma^{1} \underline{2}\right) \sigma^{1}((\lambda((\lambda \underline{2}) \underline{2})) \underline{1})$. We recursively define the following:

$$
u_{n+1}:=\mathrm{S}\left(1, \mathrm{~S}(1, \mathrm{P}(2,1,2), \mathrm{P}(2,0,1)), u_{n}\right) \text { if } n \geq 0 .
$$

The following steps are:

\begin{tabular}{||c|c|l||}
\hline \hline STEP & RULE & POSITION \\
\hline 7 & 1 & 1 \\
8 & 4 & 111 \\
9 & 10 & 11 \\
10 & 11 & 11 \\
11 & 8 & 1 \\
\hline
\end{tabular}

The current term, $\mathrm{L}\left(\mathrm{S}\left(1, \mathrm{~S}\left(2, \mathrm{P}(2,1,2), u_{0}\right), \mathrm{S}\left(1, \mathrm{P}(2,0, \mathrm{~A}(\mathrm{~L}(\mathrm{~A}(\mathrm{~L}(2), 2)), 1)), u_{0}\right)\right)\right)$ has the term $\mathrm{S}\left(1, \mathrm{P}(2,0, \mathrm{~A}(\mathrm{~L}(\mathrm{~A}(\mathrm{~L}(2), 2)), 1)), u_{0}\right)$ as a sub-term, which is written as $\left(\varphi_{0}^{2}((\lambda((\lambda \underline{2}) \underline{2})) \underline{1}) \sigma^{1} u_{0}\right)$ in the language of the $\lambda s_{e}$-calculus. This sub-term is important in the characterisation of the infinite reduction.

The following steps are given in the next table:

\begin{tabular}{||c|c|l||}
\hline \hline STEP & RULE & POSITION \\
\hline 12 & 6 & 121 \\
13 & 5 & 1211 \\
14 & 6 & 12111 \\
15 & 5 & 121111 \\
16 & 7 & 1211111 \\
17 & 1 & 121 \\
18 & 3 & 121 \\
19 & 2 & 1211 \\
20 & 3 & 12 \\
21 & 2 & 121 \\
22 & 1 & 12 \\
23 & 4 & 1211 \\
24 & 10 & 121 \\
25 & 11 & 121 \\
26 & 8 & 12 \\
\hline \hline
\end{tabular}


The current term contains the sub-term $\mathrm{S}\left(1, \mathrm{P}\left(2,0, u_{0}\right), u_{1}\right)$ which can be reduced to

$$
\mathrm{S}\left(1, \mathrm{P}(2,0, \mathrm{~A}(\mathrm{~L}(\mathrm{~A}(\mathrm{~L}(2), 2)), 1)), u_{1}\right)
$$

according to the table:

\begin{tabular}{||c|c|l||}
\hline \hline STEP & RULE & POSITION \\
\hline 27 & 11 & 1221 \\
28 & 8 & 122 \\
\hline \hline
\end{tabular}

The current term contains $\mathrm{S}\left(1, \mathrm{P}(2,0, \mathrm{~A}(\mathrm{~L}(\mathrm{~A}(\mathrm{~L}(2), 2)), 1)), u_{1}\right)$ as a sub-term which completes the first cycle of our infinite reduction. Note that we do not have a loop in these reductions because the original term is never reached again. In fact, the adequate combination of the associated calculus, named $s_{e}$, with the $\sigma$-generation rule permits one to start new simulations of $\beta$-reduction without finishing previous started simulations of $\beta$-reduction which suggests a kind of cycle. The same happens in the $\lambda \sigma$-calculus.

The next cycle is completed when the sub-term

$$
\mathrm{S}\left(1, \mathrm{P}(2,0, \mathrm{~A}(\mathrm{~L}(\mathrm{~A}(\mathrm{~L}(2), 2)), 1)), u_{2}\right)
$$

is generated. This is done by repeating the same steps from 12 to 26 in adequate positions. Additional applications of the rules 11 and 8 (in this order) will be necessary to rewrite terms of the form $\mathrm{S}\left(1, \mathrm{P}\left(2,0, u_{m}\right), u_{n}\right)$ as terms containing sub-terms of the form $\mathrm{S}\left(1, \mathrm{P}(2,0, \mathrm{~A}(\mathrm{~L}(\mathrm{~A}(\mathrm{~L}(2), 2)), 1)), u_{n}\right)$, for $n, m \geq 0$ (cf. [Gui00]). The next table presents the necessary steps to complete the second cycle:

\begin{tabular}{||c|c|l||}
\hline \hline STEP & RULE & POSITION \\
\hline 29 & 6 & 12221 \\
30 & 5 & 122211 \\
31 & 6 & 1222111 \\
32 & 5 & 12221111 \\
33 & 7 & 122211111 \\
34 & 1 & 12221 \\
35 & 3 & 12221 \\
36 & 2 & 122211 \\
37 & 3 & 1222 \\
38 & 2 & 12221 \\
39 & 1 & 1222 \\
40 & 4 & 122211 \\
41 & 10 & 12221 \\
42 & 11 & 12221 \\
43 & 8 & 1222 \\
\hline \hline
\end{tabular}

Now we need to reduce the sub-term $\mathrm{S}\left(1, \mathrm{P}\left(2,0, u_{1}\right), u_{2}\right)$ to a new term having $\mathrm{S}\left(1, \mathrm{P}\left(2,0, u_{0}\right), u_{2}\right)$ as a sub-term, from which we get the sub-term $\mathrm{S}\left(1, \mathrm{P}(2,0, \mathrm{~A}(\mathrm{~L}(\mathrm{~A}(\mathrm{~L}(2), 2)), 1)), u_{2}\right)$. The next table includes this reduction:

\begin{tabular}{||c|c|l||}
\hline \hline STEP & RULE & POSITION \\
\hline 44 & 11 & 122221 \\
45 & 8 & 12222 \\
46 & 11 & 1222221 \\
47 & 8 & 122222 \\
\hline \hline
\end{tabular}

Note that here, two applications of rules 11 and 8 (in this order) were necessary as is shown in the previous table. To continue the derivation and generate the sub-term S $\left(1, \mathrm{P}(2,0, \mathrm{~A}(\mathrm{~L}(\mathrm{~A}(\mathrm{~L}(2), 2)), 1)), u_{3}\right)$ repeat steps 29 to 47 on the adequate positions. Do not forget that additional applications of rules 11 and 


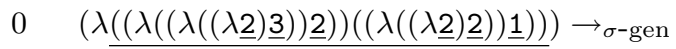

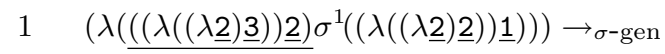

$2 \quad\left(\lambda\left(\underline{\left(((\lambda \underline{2}) \underline{3}) \sigma^{1} \underline{2}\right)} \sigma^{1}((\lambda((\lambda \underline{2}) \underline{2})) \underline{1})\right)\right) \rightarrow_{\sigma-\text { app }}$

$3 \quad\left(\lambda\left(\left(\underline{\left((\lambda \underline{2}) \sigma^{1} \underline{2}\right)}\left(\underline{3} \sigma^{1} \underline{2}\right)\right) \sigma^{1}((\lambda((\lambda \underline{2}) \underline{2})) \underline{1})\right)\right) \rightarrow \sigma \lambda$

$4 \quad\left(\lambda \underline{\left.\left(\left(\left(\lambda\left(\underline{2} \sigma^{2} \underline{2}\right)\right)\left(\underline{3} \sigma^{1} \underline{2}\right)\right) \sigma^{1}((\lambda((\lambda \underline{2}) \underline{2})) \underline{1})\right)\right) \rightarrow \sigma \text { app }}\right.$

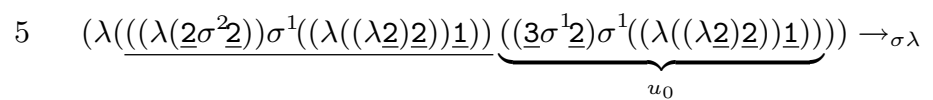

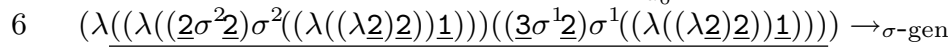

$7 \quad\left(\lambda\left(\left(\underline{\left.\underline{2} \sigma^{2} \underline{2}\right)} \sigma^{2}((\lambda((\lambda \underline{2}) \underline{2})) \underline{1})\right) \sigma^{1}\left(\left(\underline{3} \sigma^{1} \underline{2}\right) \sigma^{1}((\lambda((\lambda \underline{2}) \underline{2})) \underline{1})\right)\right)\right) \rightarrow_{\sigma \text {-des }}$

$8 \quad\left(\lambda\left(\left(\varphi_{0}^{2}(\underline{2}) \sigma^{2}((\lambda((\lambda \underline{2}) \underline{2})) \underline{1})\right) \sigma^{1}\left(\left(\underline{3} \sigma^{1} \underline{2}\right) \sigma^{1}((\lambda((\lambda \underline{2}) \underline{2})) \underline{1})\right)\right)\right) \rightarrow_{\sigma \varphi 2}$

$9 \quad\left(\lambda\left(\underline{\varphi_{0}^{2}\left(\left(\underline{2} \sigma^{1}((\lambda((\lambda \underline{2}) \underline{2})) \underline{1})\right)\right)} \sigma^{1}\left(\left(\underline{3} \sigma^{1} \underline{2}\right) \sigma^{1}((\lambda((\lambda \underline{2}) \underline{2})) \underline{1})\right)\right)\right) \rightarrow_{\varphi \sigma}$

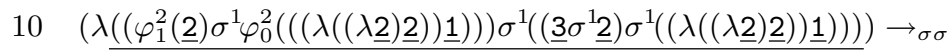

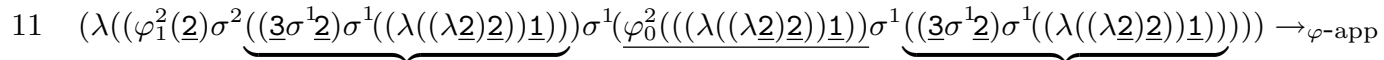

$12\left(\lambda\left(\left(\varphi_{1}^{2}(\underline{2}) \sigma^{2}\left(\left(\underline{3} \sigma^{1} \underline{2}\right) \sigma^{1}((\lambda((\lambda \underline{2}) \underline{2})) \underline{1})\right)\right) \sigma^{1}\left(\left(\underline{\varphi_{0}^{2}((\lambda((\lambda \underline{2}) \underline{2})))} \varphi_{0}^{2}(\underline{1})\right) \sigma^{1}\left(\left(\underline{3} \sigma^{1} \underline{2}\right) \sigma^{u_{0}}((\lambda((\lambda \underline{2}) \underline{2})) \underline{1})\right)\right)\right)\right) \rightarrow \varphi \lambda$

$13\left(\lambda\left(\left(\varphi_{1}^{2}(\underline{2}) \sigma^{2}\left(\left(\underline{3} \sigma^{1} \underline{2}\right) \sigma^{1}((\lambda((\lambda \underline{2}) \underline{2})) \underline{1})\right)\right) \sigma^{1}\left(\left(\left(\lambda \underline{\left.\varphi_{1}^{2}(((\lambda \underline{2}) \underline{2}))\right)} \varphi_{0}^{2}(\underline{1})\right) \sigma^{1}\left(\left(\underline{3} \sigma^{1} \underline{2}\right) \sigma^{1}((\lambda((\lambda \underline{2}) \underline{2})) \underline{1})\right)\right)\right)\right) \rightarrow \varphi\right.$-app

$14\left(\lambda\left(\left(\varphi_{1}^{2}(\underline{2}) \sigma^{2}\left(\left(\underline{3} \sigma^{1} \underline{2}\right) \sigma^{1}((\lambda((\lambda \underline{2}) \underline{2})) \underline{1})\right)\right) \sigma^{1}\left(\left(\left(\lambda\left(\underline{\varphi_{1}^{2}((\lambda \underline{2}))} \varphi_{1}^{2}(\underline{2})\right)\right) \varphi_{0}^{2}(\underline{1})\right) \sigma^{1}\left(\left(\underline{3} \sigma^{1} \underline{2}\right) \sigma^{1}((\lambda((\lambda \underline{2}) \underline{2})) \underline{1})\right)\right)\right)\right) \rightarrow \varphi \lambda$

$15\left(\lambda\left(\left(\varphi_{1}^{2}(\underline{2}) \sigma^{2}\left(\left(\underline{3} \sigma^{1} \underline{2}\right) \sigma^{1}((\lambda((\lambda \underline{2}) \underline{2})) \underline{1})\right)\right) \sigma^{1}\left(\left(\left(\lambda\left(\left(\lambda \underline{\left.\varphi_{2}^{2}(\underline{2})\right)}\right) \varphi_{1}^{2}(\underline{2})\right)\right) \varphi_{0}^{2}(\underline{1})\right) \sigma^{1}\left(\left(\underline{3} \sigma^{1} \underline{2}\right) \sigma^{1}((\lambda((\lambda \underline{2}) \underline{2})) \underline{1})\right)\right)\right)\right) \rightarrow \varphi$-des

$16\left(\lambda\left(\left(\varphi_{1}^{2}(\underline{2}) \sigma^{2}\left(\left(\underline{3} \sigma^{1} \underline{2}\right) \sigma^{1}((\lambda((\lambda \underline{2}) \underline{2})) \underline{1})\right)\right) \sigma^{1}\left(\left(\left(\lambda\left((\lambda \underline{2}) \varphi_{1}^{2}(\underline{2})\right)\right) \varphi_{0}^{2}(\underline{1})\right) \sigma^{1}\left(\left(\underline{3} \sigma^{1} \underline{2}\right) \sigma^{1}((\lambda((\lambda \underline{2}) \underline{2})) \underline{1})\right)\right)\right)\right) \rightarrow \sigma$-gen

$17\left(\lambda\left(\left(\varphi_{1}^{2}(\underline{2}) \sigma^{2}\left(\left(\underline{3} \sigma^{1} \underline{2}\right) \sigma^{1}((\lambda((\lambda \underline{2}) \underline{2})) \underline{1})\right)\right) \sigma^{1}\left(\left(\left((\lambda \underline{2}) \varphi_{1}^{2}(\underline{2})\right) \sigma^{1} \varphi_{0}^{2}(\underline{1})\right) \sigma^{1}\left(\left(\underline{3}^{1} \underline{2}\right) \sigma^{1}((\lambda((\lambda \underline{2}) \underline{2})) \underline{1})\right)\right)\right)\right) \rightarrow \sigma-$ app

$18\left(\lambda\left(\left(\varphi_{1}^{2}(\underline{2}) \sigma^{2}\left(\left(\underline{3} \sigma^{1} \underline{2}\right) \sigma^{1}((\lambda((\lambda \underline{2}) \underline{2})) \underline{1})\right)\right) \sigma^{1}\left(\left(\left((\lambda \underline{2}) \sigma^{1} \varphi_{0}^{2}(\underline{1})\right)\left(\varphi_{1}^{2}(\underline{2}) \sigma^{1} \varphi_{0}^{2}(\underline{1})\right)\right) \sigma^{1}\left(\left(\underline{3} \sigma^{1} \underline{2}\right) \sigma^{1}((\lambda((\lambda \underline{2}) \underline{2})) \underline{1})\right)\right)\right)\right) \rightarrow \sigma \lambda$

$19\left(\lambda\left(\left(\varphi_{1}^{2}(\underline{2}) \sigma^{2}\left(\left(\underline{3} \sigma^{1} \underline{2}\right) \sigma^{1}((\lambda((\lambda \underline{2}) \underline{2})) \underline{1})\right)\right) \sigma^{1}\left(\left(\left(\lambda\left(\underline{2} \sigma^{2} \varphi_{0}^{2}(\underline{1})\right)\right)\left(\varphi_{1}^{2}(\underline{2}) \sigma^{1} \varphi_{0}^{2}(\underline{1})\right)\right) \sigma^{1}\left(\left(\underline{3} \sigma^{1} \underline{2}\right) \sigma^{1}((\lambda((\lambda \underline{2}) \underline{2})) \underline{1})\right)\right)\right) \rightarrow \sigma\right.$-app

$20 \quad\left(\lambda\left(\left(\varphi_{1}^{2}(\underline{2}) \sigma^{2}\left(\left(\underline{3} \sigma^{1} \underline{2}\right) \sigma^{1}((\lambda((\lambda \underline{2}) \underline{2})) \underline{1})\right)\right) \sigma^{1}\left(\left(\left(\lambda\left(\underline{2} \sigma^{2} \varphi_{0}^{2}(\underline{1})\right)\right) \sigma^{1}\left(\left(\underline{3} \sigma^{1} \underline{2}\right) \sigma^{1}((\lambda((\lambda \underline{2}) \underline{2})) \underline{1})\right)\right)\right.\right.\right.$ $\left.\left.\left.\left(\left(\varphi_{1}^{2}(\underline{2}) \sigma^{1} \varphi_{0}^{2}(\underline{1})\right) \sigma^{1}\left(\left(\underline{3} \sigma^{1} \underline{2}\right) \sigma^{1}((\lambda((\lambda \underline{2}) \underline{2})) \underline{1})\right)\right)\right)\right)\right) \rightarrow \sigma \lambda$

$21 \quad\left(\lambda\left(\left(\varphi_{1}^{2}(\underline{2}) \sigma^{2}\left(\left(\underline{3} \sigma^{1} \underline{2}\right) \sigma^{1}((\lambda((\lambda \underline{2}) \underline{2})) \underline{1})\right)\right) \sigma^{1}\right.\right.$ $\underline{\left.\left.\left(\left(\lambda\left(\left(\underline{2} \sigma^{2} \varphi_{0}^{2}(\underline{1})\right) \sigma^{2}\left(\left(\underline{3} \sigma^{1} \underline{2}\right) \sigma^{1}((\lambda((\lambda \underline{2}) \underline{2})) \underline{1})\right)\right)\right)\left(\left(\varphi_{1}^{2}(\underline{2}) \sigma^{1} \varphi_{0}^{2}(\underline{1})\right) \sigma^{1}\left(\left(\underline{3} \sigma^{1} \underline{2}\right) \sigma^{1}((\lambda((\lambda \underline{2}) \underline{2})) \underline{1})\right)\right)\right)\right)\right) \rightarrow \sigma-\text { gen }}$

$22 \quad\left(\lambda\left(\left(\varphi_{1}^{2}(\underline{2}) \sigma^{2}\left(\left(\underline{3} \sigma^{1} \underline{2}\right) \sigma^{1}((\lambda((\lambda \underline{2}) \underline{2})) \underline{1})\right)\right) \sigma^{1}\left(\left(\left(\underline{2} \sigma^{2} \varphi_{0}^{2}(\underline{1})\right) \sigma^{2}\left(\left(\underline{3} \sigma^{1} \underline{2}\right) \sigma^{1}\right.\right.\right.\right.\right.$ $\left.\left.\left.((\lambda((\lambda \underline{2}) \underline{2})) \underline{1}))) \sigma^{1}\left(\left(\varphi_{1}^{2}(\underline{2}) \sigma^{1} \varphi_{0}^{2}(\underline{1})\right) \sigma^{1}\left(\left(\underline{3} \sigma^{\underline{2}} \underline{2}\right) \sigma^{1}((\lambda((\lambda \underline{2}) \underline{2})) \underline{1})\right)\right)\right)\right)\right) \rightarrow \sigma$-des

$23\left(\lambda\left(\left(\varphi_{1}^{2}(\underline{2}) \sigma^{2}\left(\left(\underline{3} \sigma^{1} \underline{2}\right) \sigma^{1}((\lambda((\lambda \underline{2}) \underline{2})) \underline{1})\right)\right) \sigma^{1}\left(\left(\varphi_{0}^{2}\left(\varphi_{0}^{2}(\underline{1})\right) \sigma^{2}\left(\left(\underline{3} \sigma^{1} \underline{2}\right) \sigma^{1}((\lambda((\lambda \underline{2}) \underline{2})) \underline{1})\right)\right) \sigma^{1}\right.\right.\right.$ $\left.\left.\left.\left(\left(\varphi_{1}^{2}(\underline{2}) \sigma^{1} \varphi_{0}^{2}(\underline{1})\right) \sigma^{1}\left(\left(\underline{3} \sigma^{1} \underline{2}\right) \sigma^{1}((\lambda((\lambda \underline{2}) \underline{2})) \underline{1})\right)\right)\right)\right)\right) \rightarrow \sigma \varphi 2$

$24 \quad\left(\lambda\left(\left(\varphi_{1}^{2}(\underline{2}) \sigma^{2}\left(\left(\underline{3} \sigma^{1} \underline{2}\right) \sigma^{1}((\lambda((\lambda \underline{2}) \underline{2})) \underline{1})\right)\right) \sigma^{1}\left(\varphi_{0}^{2}\left(\left(\varphi_{0}^{2}(\underline{1}) \sigma^{1}\left(\left(\underline{3} \sigma^{1} \underline{2}\right) \sigma^{1}((\lambda((\lambda \underline{2}) \underline{2})) \underline{1})\right)\right)\right) \sigma^{1}\right.\right.\right.$ $\left.\left.\left.\left(\left(\varphi_{1}^{2}(\underline{2}) \sigma^{1} \varphi_{0}^{2}(\underline{1})\right) \sigma^{1}\left(\left(\underline{3} \sigma^{1} \underline{2}\right) \sigma^{1}((\lambda((\lambda \underline{2}) \underline{2})) \underline{1})\right)\right)\right)\right)\right) \rightarrow \varphi \sigma$

$25 \quad\left(\lambda\left(\left(\varphi_{1}^{2}(\underline{2}) \sigma^{2}\left(\left(\underline{3} \sigma^{1} \underline{2}\right) \sigma^{1}((\lambda((\lambda \underline{2}) \underline{2})) \underline{1})\right)\right) \sigma^{1}\right.\right.$

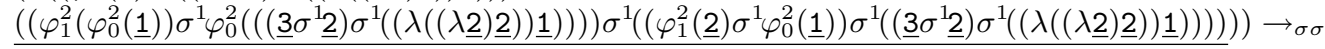

$26 \quad\left(\lambda\left(\left(\varphi_{1}^{2}(\underline{2}) \sigma^{2}\left(\left(\underline{3} \sigma^{1} \underline{2}\right) \sigma^{1}((\lambda((\lambda \underline{2}) \underline{2})) \underline{1})\right)\right) \sigma^{1}\left(\left(\varphi_{1}^{2}\left(\varphi_{0}^{2}(\underline{1})\right) \sigma^{2}\left(\left(\varphi_{1}^{2}(\underline{2}) \sigma^{1} \varphi_{0}^{2}(\underline{1})\right) \sigma^{1}\left(\left(\underline{3} \sigma^{1} \underline{2}\right) \sigma^{1}\right.\right.\right.\right.\right.\right.$

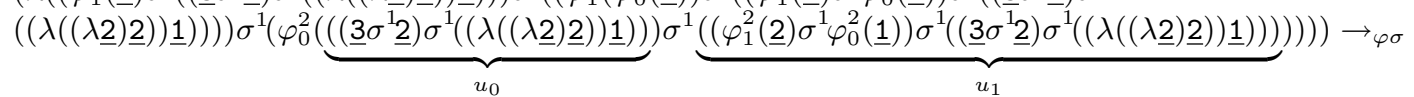

$27 \quad\left(\lambda\left(\left(\varphi_{1}^{2}(\underline{2}) \sigma^{2}\left(\left(\underline{3} \sigma^{1} \underline{2}\right) \sigma^{1}((\lambda((\lambda \underline{2}) \underline{2})) \underline{1})\right)\right) \sigma^{1}\left(\left(\varphi_{1}^{2}\left(\varphi_{0}^{2}(\underline{1})\right) \sigma^{2}\left(\left(\varphi_{1}^{2}(\underline{2}) \sigma^{1} \varphi_{0}^{2}(\underline{1})\right) \sigma^{1}\left(\left(\underline{3} \sigma^{1} \underline{2}\right) \sigma^{1}\right.\right.\right.\right.\right.\right.$ $\left.\left.((\lambda((\lambda \underline{2}) \underline{2})) \underline{1})))) \sigma^{1}\left(\left(\varphi_{1}^{2}\left(\left(\underline{3} \sigma^{1} \underline{2}\right)\right) \sigma^{1} \varphi_{0}^{2}(((\lambda((\lambda \underline{2}) \underline{2})) \underline{1}))\right) \sigma^{1}\left(\left(\varphi_{1}^{2}(\underline{2}) \sigma^{1} \varphi_{0}^{2}(\underline{1})\right) \sigma^{1}\left(\left(\underline{3} \sigma^{1} \underline{2}\right) \sigma^{1}((\lambda((\lambda \underline{2}) \underline{2})) \underline{1})\right)\right)\right)\right)\right) \rightarrow \sigma \sigma$

$28\left(\lambda\left(\left(\varphi_{1}^{2}(\underline{2}) \sigma^{2}\left(\left(\underline{3} \sigma^{1} \underline{2}\right) \sigma^{1}((\lambda((\lambda \underline{2}) \underline{2})) \underline{1})\right)\right) \sigma^{1}\left(\left(\varphi_{1}^{2}\left(\varphi_{0}^{2}(\underline{1})\right) \sigma^{2}\left(\left(\varphi_{1}^{2}(\underline{2}) \sigma^{1} \varphi_{0}^{2}(\underline{1})\right) \sigma^{1}\left(\left(\underline{3} \sigma^{1} \underline{2}\right) \sigma^{1}\right.\right.\right.\right.\right.\right.$ $((\lambda((\lambda \underline{2}) \underline{2})) \underline{1})))) \sigma^{1}\left(\left(\varphi_{1}^{2}\left(\left(\underline{3} \sigma^{1} \underline{2}\right)\right) \sigma^{2}\left(\left(\varphi_{1}^{2}(\underline{2}) \sigma^{1} \varphi_{0}^{2}(\underline{1})\right) \sigma^{1}\left(\left(\underline{3} \sigma^{1} \underline{2}\right) \sigma^{1}((\lambda((\lambda \underline{2}) \underline{2})) \underline{1})\right)\right)\right) \sigma^{1} \varphi_{0}^{2}(((\lambda((\lambda \underline{2}) \underline{2})) \underline{1})) \sigma^{1}\right.$ $\left.\left.\left.\left.\left(\left(\varphi_{1}^{2}(\underline{2}) \sigma^{1} \varphi_{0}^{2}(\underline{1})\right) \sigma^{1}\left(\left(\underline{3} \sigma^{1} \underline{2}\right) \sigma^{1}((\lambda((\lambda \underline{2}) \underline{2})) \underline{1})\right)\right)\right)\right)\right)\right) \quad 16$

$u_{1}$

Table 2: The counter-example of Guillaume 
8 will be necessary. The shape of the infinite derivation can be written as

$\lambda((\lambda((\lambda((\lambda \underline{2}) \underline{3})) \underline{2}))(\lambda((\lambda \underline{2}) \underline{2})) \underline{1}) \rightsquigarrow$

$\left(\varphi_{0}^{2}((\lambda((\lambda \underline{2}) \underline{2})) \underline{1}) \sigma^{1} u_{0}\right) \rightsquigarrow$

$\left(\varphi_{0}^{2}((\lambda((\lambda \underline{2}) \underline{2})) \underline{1}) \sigma^{1} u_{1}\right) \rightsquigarrow$

$\left(\varphi_{0}^{2}((\lambda((\lambda \underline{2}) \underline{2})) \underline{1}) \sigma^{1} u_{2}\right) \rightsquigarrow \cdots$

where $\rightsquigarrow$ means "leads to a term containing the following expression as a sub-term".

The latex output of the first 28 steps of the infinite derivation can be automatically generated. To do so, type the prompt shell command

./subsexpl.bin guillaume (or ./ledit.out ./subsexpl.bin guillaume)

where guillaume is a file distributed with the source code of SUBSEXPL ${ }^{5}$. The system will generate the guillaume-lse.dvi file. The result is shown in the next page which includes the number of the steps and some subscripts for ease of reading, such as $u_{0}$ and $u_{1}$, that do not appear in the latex output generated by the system SUBSEXPL. In this example, the latex code of the output can be found in the guillaume-lse file.

The animated generation of the initial steps of these infinite derivations inside each calculus gives an easy-to-understand and more intuitive insight as to why the PSN property fails. In fact, for understanding these counter-examples (and their importance) directly from the related papers ([Mel95, Gui00]), the reader needs to follow a sequence of inductively proved lemmas and theorems. This is of course necessary for an adequate formalisation of this fact, but starting from this approach presumes previous knowledge about what PSN means and we believe that in this way the reader may lose the focus about the mechanics of these infinite derivations in the associated substitution calculi. Even worst, the reader may lose a very important aspect: namely, the meaning and the implications of losing PSN in these calculi. Consequently, we believe SUBSEXPL is an adequate and useful tool for intuitively understanding the details and difficulties concerned with this and other general properties of the $\lambda$-calculus as well as of explicit substitutions calculi.

In [Nad99, LN02] it has been conjectured that PSN holds in $\lambda_{\text {susp }}$. But until now, there is neither a formal proof nor a counter-example of this conjecture. We believe that SUBSEXPL may act as an adequate tool for reasoning about open questions like this since, every reduction (either from a pure or a non pure $\lambda$-term) can be simulated in an easy, fast and secure way in this system.

\section{Conclusions and future work}

We presented the system SUBSEXPL which is an Ocaml implementation of the rewriting rules of the $\lambda \sigma$, the $\lambda s_{e}$ and the suspension calculi of explicit substitutions, although according to the current structure the inclusion of other explicit substitutions calculi can be easily done.

We showed how the system has been applied both to educational and research purposes. Its educational use includes:

- the visualisation of the computational adequacy of the $\lambda$-calculus via specification of numerical functions and programming operators;

- the visualisation of (non trivial) properties of the $\lambda$-calculus such as non terminality and the normalisation theorem;

- the illustration of the problem of implicitness of the substitution operator and how this is resolved in real implementations by explicit substitutions calculi; etc.

To give to students a flavor of the computational power of the $\lambda$-calculus, we showed how the system can assist in building computational operators such as iteration, conditionals and repetitions and functions such as the factorial function. Its research applications includes:

- analysis of non trivial properties of explicit substitutions calculi;

${ }^{5}$ See www . mat.unb.br/ ayala/TCgroup 
- comparing calculi of explicit substitutions.

The former was illustrated by showing how one can check the proofs of Melliès and Guillaume of the fact that neither $\lambda \sigma$ nor $\lambda s_{e}$ preserve strong normalisation. The latter by showing how the system assisted us in the proof that $\lambda s_{e}$ is more efficient than the suspension calculus and is incomparable to the $\lambda \sigma$-calculus in the simulation of one-step $\beta$-reduction [AMK05].

Furthermore, SUBSEXPL gives correct implementations of $\eta$-reduction for each of the three explicit substitutions calculi treated here. For the $\lambda s_{e}$-calculus this implementation is also clean, but for $\lambda \sigma$ and $\lambda_{\text {SusP }}$ (and by the nature of these calculi), the simulation of one-step $\eta$-reduction requires the use of rewriting rules that are not strictly related to this one-step simulation.

Other authors have presented tools which allow for manipulation of $\lambda$-expressions in a similar way; for example Huet presented a tool and illustrated how this can be applied for assisting the understanding of non trivial properties of the $\lambda$-calculus such as Böhm's theorem [Hu93]. The novelty of SUBSEXPL with relation to these applications is that it follows the de Bruijn philosophy of avoiding names, which makes our tool also adequate for assisting the reasoning about properties of explicit substitution calculi.

As any modern computational system, SUBSEXPL is in constant development and new features should be included in future versions. Among these features, we can point out the inclusion of variations of the suspension calculus that combine applications of $\beta$-reduction and the development of new modules for dealing with simply typed $\lambda$-terms and $\lambda$-calculus with names. Moreover, we will develop an EMACS mode which may ease the inclusion of some common structures used to build more complex terms such as, the factorial function studied in subsection 3.1.

Acknowledgments. We would like to thank Manuel Maarek and Stéphane Gimenez for the useful help with Ocaml and suggestions to improve the system, and to Marrise Neves da Rocha for the development of many examples with SUBSEXPL.

\section{References}

[ACCL91] M. Abadi, L. Cardelli, P.-L. Curien, and J.-J. Lévy. Explicit Substitutions. JFP, 1(4):375-416, 1991.

[AMK05] M. Ayala-Rincón, F.L.C. de Moura and F. Kamareddine. Comparing and Implementing Calculi of Explicit Substitutions with Eta-Reduction. To appear in WoLLIC'02 Special Issue of Annals of Pure and Applied Logic, 2005.

[ARK01] M. Ayala-Rincón and F. Kamareddine. Unification via the $\lambda s_{e}$-Style of Explicit Substitution. The Logical Journal of the Interest Group in Pure and Applied Logics, 9(4):489-523, 2001.

[Bar84] H. P. Barendregt. The Lambda Calculus : Its Syntax and Semantics (revised edition). North Holland, 1984.

[Bar92] H. P. Barendregt. A-calculi with types. In S. Abramsky, D. M. Gabbay, and T. S. E. Maibaum, editors, Background: Computational Structures, volume 2 of Handbook of Logic in Computer Science, pages 117-309, 1992.

[Bri95] D. Briaud. An explicit Eta rewrite rule. In Typed Lambda Calculi and Applications, volume 902 of LNCS, pages 94-108. Springer, 1995.

[DHK00] G. Dowek, T. Hardin, and C. Kirchner. Higher-order Unification via Explicit Substitutions. Information and Computation, 157(1/2):183-235, 2000.

[Fer05] Ferreira H., Análise de Mecanismos para Combinar passos de Beta-Contração em Cálculos de Substituições Explícitas, Master's thesis, Departamento de Matemática, Universidade de Brasília, 2005, In Portuguese. 
[Gui00] B. Guillaume. The $\lambda s_{e}$-calculus Does Not Preserve Strong Normalization. JFP, 10(4):321-325, 2000.

[Har92] T. Hardin. Eta-conversion for the languages of explicit substitutions. In Algebraic and Logic Programming, volume 632 of LNCS, pages 306-321. Springer, 1992.

[Hu93] G. Huet. An Analysis of Böhm's Theorem. TCS, 121:145-167, 1993.

[Kes00] D. Kesner. Confluence of extensional and non-extensional $\lambda$-calculi with explicit substitutions. TCS, 238(1-2):183-220, 2000.

[KN96] F. Kamareddine and R. P. Nederpelt. A useful $\lambda$-notation. TCS, 155:85-109, 1996.

[KR95] F. Kamareddine and A. Ríos. A $\lambda$-calculus à la de Bruijn with Explicit Substitutions. In Proc. Seventh Int. Symposium on Programming Languages(PLILP'95), volume 982 of LNCS, pages 45-62. Springer, 1995.

[KR00] F. Kamareddine and A. Ríos. Relating the $\lambda \sigma$ - and $\lambda s$-Styles of Explicit Substitutions. Journal of Logic and Computation, 10(3):349-380, 2000.

[LN02] C. Liang and G. Nadathur. Tradeoffs in the Intensional Representation of Lambda Terms. In S. Tison, editor, Rewriting Techniques and Applications (RTA 2002), volume 2378 of LNCS, pages 192-206. Springer-Verlag, 2002.

[LNQ04] Liang C., G.Nadathur and Qi X., Choices in Representation and Reduction Strategies for Lambda Terms in Intensional Contexts, Journal of Automated Reasoning, volume 33, number 2, pages 89-132, 2004.

[Mel95] P.-A. Melliès. Typed $\lambda$-calculi with explicit substitutions may not terminate. In Proc. 2nd Int. Conf. on Typed Lambda Calculi and Applications(TLCA'95). LNCS, 902, 1995.

[dMKAR05] F. L. C. de Moura, F. Kamareddine, and M. Ayala-Rincón. Second Order Matching via Explicit Substitutions. In Proc. of the 11th Int. Conf. on Logic for Programming Artificial Intelligence and Reasoning (LPAR-2004). Springer-Verlag LNAI, 2005.

[Nad99] G. Nadathur. A fine-grained notation for lambda terms and its use in intensional operations. J. of Func. and Logic Programming, 2:1-62, 1999.

[Nad02] G. Nadathur. The Suspension Notation for Lambda Terms and its Use in Metalanguage Implementations. In R. de Queiroz, editor, Proc. 9th Workshop on Logic, Language, Information and Computation (WoLLIC 2002), volume 67 of ENTCS. Elsevier Science Publishers, 2002.

[NaQi03] Nadathur G. AND Qi X., Explicit Substitutions in the Reduction of Lambda Terms, Fifth ACMSIGPLAN International Conference on Principles and Practice of Declarative Programming, pages 195206, 2003.

[NGdV94] R. P. Nederpelt, J. H. Geuvers, and R. C. de Vrijer. Selected papers on Automath. North-Holland, Amsterdam, 1994.

[Río93] A. Ríos. Contribution à l'étude des $\lambda$-calculs avec substitutions explicites. PhD thesis, Université de Paris 7, 1993. 